\title{
KJCCM
}

\section{Should Very Old Patients Be Admitted to the Intensive Care Units?}

\author{
Jun Kwon Cha ${ }^{1}$ and In-Ae Song ${ }^{2}$
}

${ }^{1}$ Department of Emergency Medicine, Konkuk University Chungju Hospital, Chungju; ${ }^{2}$ Department of Anesthesiology and Pain Medicine, Seoul National University Bundang Hospital, Seongnam, Korea

\section{Dear Editor:}

I read with great interest the article "Demographic Changes in Intensive Care Units in Korea over the Last Decade and Outcomes of Elderly Patients: A SingleCenter Retrospective Study" published in the Korean Journal of Critical Care Medicine in May 2017 [1]. The results indicated that the proportion of inpatients aged 65-79 years admitted to an intensive care unit (ICU) increased from $47.9 \%$ in 2005 to $63.7 \%$ in 2014 , and the proportion of ICU-hospitalized patients older than 80 years increased from $12.8 \%$ in 2005 to $20.7 \%$ in 2014 . However, the overall mortality rate did not increase despite a higher mortality rate in the elderly than in the younger patients. These results are worthy and impressively demonstrate the recent changing trends in demographic data of ICU patients in Korea. However, I would like to comment on the following two points.

First, the authors might have shown a slightly lower sepsis prevalence, not greater than $1 \%$ for each subgroup in the ICU. The study conducted by Oh et al. [2], using the Health Insurance Review \& Assessment Service database, revealed that the inhospital mortality of patients with sepsis was as high as $38.9 \%$, and the proportion of sepsis increased with age. In addition, in the United States, sepsis was ranked 11 in the top primary diagnoses in 1996 among patients older than 65 years who were admitted to the ICU, but in 2010, sepsis was ranked 1 among the primary diagnoses in older patients admitted to the ICU [3]. In Korea, where the proportion of elderly population is rapidly increasing, it is expected that the rate of primary diagnosis of sepsis in the elderly patients who are admitted to the ICU would be higher. It is considered to be a limitation due to single-center studies, and nationwide demographic studies of ICU patients are required.

Second, there had been questions regarding the appropriateness of ICU hospitalization of very old patients (VOPs) aged greater than 80 years with chronic illnesses. Roch et al. [4] reported that among patients older than 80 years, the ICU
Received on October 2, 2017

Accepted on November 27, 2017

In-Ae Song

Department of Anesthesiology and Pain Medicine, Seoul National University Bundang Hospital, 82 Gumi-ro 173beongil, Bundang-gu, Seongnam 13620, Korea Tel: +82-31-787-7499

Fax: +82-31-787-4063

E-mail: songoficu@gmail.com

*No potential conflict of interest relevant to this article was reported.

cc This is an Open Access article distributed under the terms of the Creative Commons Attribution Non-Commercial License (http://creativecommons.org/ licenses/by-nc/4.0/) which permits unrestricted non-commercial use, distribution, and reproduction in any medium, provided the original work is properly cited. Copyright (c) 2017 The Korean Society of Critical Care Medicine 
mortality rate was $46 \%$, 1-year mortality rate was $72 \%$, and 2-year mortality rate was $79 \%$. The purpose of care for VOPs admitted to ICU might be just life-sustaining therapy rather than survival with highly qualified life. Increased long life-sustaining therapy might result in increased economic and emotional stress for patients, families, and medical costs. In addition, it will be difficult to properly operate in the ICU because of the prolonged length of stay of VOPs with chronic illnesses and the shortage of human resources for the other patients.

Nguyen et al. [5] suggested that the outcome of admission to the ICU of a VOP for postoperative care after an unplanned surgery or severe medical problems would be dismal, and recommended admission to a regular ward or acute care unit rather than ICU admission. However, there is a lack of legal backgrounds for doctors and VOPs with chronic illness to make advanced plans for the proper end of life care in Korea. There have been significant concerns about a new law on hospice and palliative care and withdrawal of life-sustaining therapy, which will be implemented in 2018 because it includes ambiguous or difficult provisions to be applied in medical reality [6]. Its improvement and supplement will be required. Additionally, advanced-care planning regarding ICU admission with patients or their families seems to improve the quality of life and satisfaction level and reduce related mental stress and depression. In these cases, we need to make an early and active palliative care consultation system after ICU admission to reduce life-sustaining therapy and decrease ICU length of stay $[7,8]$.

\section{References}

1. Lim JU, Lee J, Ha JH, Kang HH, Lee SH, Moon HS. Demographic changes in intensive care units in Korea over the last decade and outcomes of elderly patients: a single-center retrospective study. Korean J Crit Care Med 2017;32:164-73.

2. Oh SY, Cho S, Lee H, Chang EJ, Min SH, Ryu HG. Sepsis in patients receiving immunosuppressive drugs in Korea: analysis of the National Insurance Database from 2009 to 2013. Korean J Crit Care Med 2015;30:249-57.

3. Sjoding MW, Prescott HC, Wunsch H, Iwashyna TJ, Cooke CR. Longitudinal changes in ICU admissions among elderly patients in the United States. Crit Care Med 2016;44:1353-60.

4. Roch A, Wiramus S, Pauly V, Forel JM, Guervilly $\mathrm{C}$, Gainnier M, et al. Long-term outcome in medical patients aged 80 or over following admission to an intensive care unit. Crit Care 2011;15:R36.

5. Nguyen YL, Angus DC, Boumendil A, Guidet B. The challenge of admitting the very elderly to intensive care. Ann Intensive Care 2011;1:29.

6. Act on hospice and palliative care and withdrawal of a life-sustaining therapy for dying patients, No. 14013 (Feb 3, 2016).

7. Detering KM, Hancock AD, Reade MC, Silvester W. The impact of advance care planning on end of life care in elderly patients: randomised controlled trial. BMJ 2010;340:c1345.

8. Zalenski RJ, Jones SS, Courage C, Waselewsky DR, Kostaroff AS, Kaufman D, et al. Impact of palliative care screening and consultation in the ICU: a multihospital quality improvement project. J Pain Symptom Manage 2017;53:5-12.e3.

\section{The Authors Reply}

We appreciate your interest in our paper and are thankful for taking the time to express your opinions. We would also like to thank you for the opportunity to clarify aspects of our methodology in relation to concerns on diagnostic criteria of the study patients and further express our opinions on the issue of elderly patients' intensive care unit (ICU) care.

As for the low proportion of sepsis, the article has a limitation. The Materials and Method section states that "The ICU patients with diagnoses associated with ICU mortality were classified into 10 subcategories. Diagno- 